\title{
CONVERGENCE RATE IN MULTIDIMENSIONAL IRREGULAR SAMPLING RESTORATION
}

\author{
Andriy Ya. Olenko And Tibor K. PogÁny
}

Abstract. New magnitude of truncation error upper bound and convergence rate are obtained for Whittaker-Kotel'nikov-Shannon (WKS) sampling restoration sum for Bernstein function class $B_{\pi, d}^{q}, q \geqslant 1, d \in \mathbb{N}$, when the sampled functions decay rate is unknown. The case of multidimensional irregular sampling is discussed.

Mathematics subject classification (2000): Primary 94A20, 26D15; Secondary 30D15, 41A05.

Keywords and phrases: Whittaker-Kotel'nikov-Shannon sampling restoration formula; approximation/interpolation error level; truncation error upper bound; irregular sampling; multidimensional sampling; convergence rate.

\section{REFERENCES}

[1] Flornes K.M., Lyubars KiI Yu. AND SeIP K., A direct interpolation method for irregular sampling, Appl. Comput. Harmon. Anal., 8, 1 (2000), 113-121.

[2] HAN Y.-S., Plancherel-Pólya type inequality on spaces of homogeneous type and its applications, Proc. Amer. Math. Soc., 126, 11 (1998), 3315-3327.

[3] Higgins J. R., Sampling in Fourier and Signal Analysis: Foundations, Clarendon Press, Oxford, 1996.

[4] Hinsen G., Irregular sampling of bandlimited $L^{p}$-functions, J. Approx. Theory, 72 (1993), 346-364.

[5] HinsEN G. AND KLÖSTERS D., The sampling series as a limiting case of Lagrange interpolation, Appl. Anal., 49 (1993), 49-60.

[6] Marvasti F., Ed., Nonuniform Sampling: Theory and Practice, Kluwer Academic Publishers, Boston, 2001.

[7] NikOLSKII S. M., Approximation of functions of several variables and imbedding theorems, SpringerVerlag, New York, 1975.

[8] Olenko A. Ya. And Pogány T. K., Direct Lagrange-Yen Type Interpolation of Random Fields, Theor. Stoch. Proc., 9 (25), 3-4 (2003), 242-254.

[9] Olenko A. YA. And PogÁNY T. K., Sharp exact upper bound for interpolation remainder of random processes, Theor. Probab. Math. Statist., 71 (2004), 133-144.

[10] Olenko A. YA. And PogÁny T. K., On sharp exact bounds for remainders in multidimensional sampling theorem, Sampl. Theory Signal Image Process, 6, 3 (2007), 249-272.

[11] Olenko A. YA. AND PogÁNy T. K., Universal truncation error upper bounds in sampling restoration, Georgian Math. J. (to appear).

[12] Olenko A. YA. AND PogÁNY T. K., Universal truncation error upper bounds in irregular sampling restoration, Appl. Anal. (to appear).

[13] Pesenson I., Plancherel-Pólya type inequalites for entire functions of exponential type in $L_{p}\left(\mathbb{R}^{d}\right)$, J. Math. Anal. Appl., 330, 2 (2007), 1194-1206.

[14] Plancherel M. and Pólya Gy, Fonctions entières et intégrales de Fourier multiples II, Comment. Math. Helv., 10 (1937), 110-163.

[15] PogÁny T. K., Multidimensional Lagrange-Yen interpolation via Kotel'nikov-Shannon sampling formulas, Ukr. Math. J., 50, 11 (2003), 1810-1827.

[16] QI FENG, A class of logarithmically completely monotonic functions and the best bounds in the first Kershaw's double inequality, J. Comput. and Applied Math., 206, 2 (2007), 1007-1014. 
[17] QI FENG, Bounds for the ratio of two Gamma functions, RGMIA Res. Rep. Coll., 11, 3 (2008), 1-63.

[18] TRIEBEL H., Theory of Function spaces, II, Birkhäuser Verlag, Basel, 1992.

[19] Voss J. J., Irregular sampling: error analysis, applications, and extensions, In CD-ROM Nonuniform Sampling: Theory and Practice, Kluwer Academic Publishers, Boston, 2001.

[20] Wendel J., Note on the gamma function, Amer. Math. Monthly, 55 (1948), 563-564.

[21] YEN J. L., On nonuniform sampling of bandwidth limited signals, IRE Trans. Circuit Theory, CT-3 (1956), 251-257. 